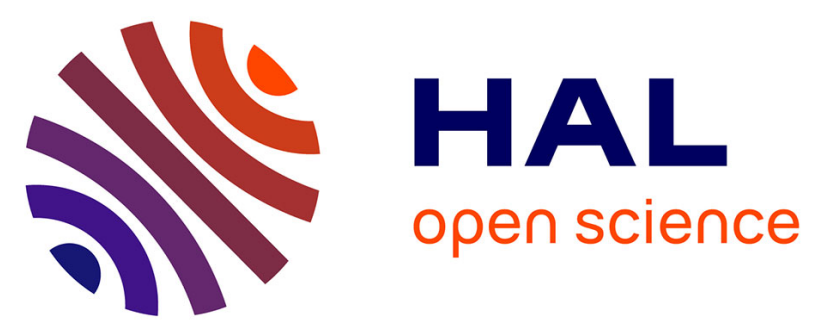

\title{
Kinematic design of a translational parallel mechanism based on sub-kinematic chain determined workspace superposition
}

\author{
Huiping Shen, Yinan Zhao, Guanglei Wu, Ju Li, Damien Chablat
}

\section{- To cite this version:}

Huiping Shen, Yinan Zhao, Guanglei Wu, Ju Li, Damien Chablat. Kinematic design of a translational parallel mechanism based on sub-kinematic chain determined workspace superposition. Proceedings of the Institution of Mechanical Engineers, Part C: Journal of Mechanical Engineering Science, 2021, pp.095440622110046. 10.1177/09544062211004653 . hal-03241640

\author{
HAL Id: hal-03241640 \\ https://hal.science/hal-03241640
}

Submitted on 2 Jun 2021

HAL is a multi-disciplinary open access archive for the deposit and dissemination of scientific research documents, whether they are published or not. The documents may come from teaching and research institutions in France or abroad, or from public or private research centers.
L'archive ouverte pluridisciplinaire HAL, est destinée au dépôt et à la diffusion de documents scientifiques de niveau recherche, publiés ou non, émanant des établissements d'enseignement et de recherche français ou étrangers, des laboratoires publics ou privés. 


\title{
Kinematic design of a translational parallel mechanism based on sub-kinematic chain determined workspace superposition
}

\author{
Huiping Shen ${ }^{1}$, Yinan $\mathrm{Zhao}^{1}$, Guanglei $\mathrm{Wu}^{* 2}$, Ju Li ${ }^{1}$, Damien Chablat ${ }^{3}$ \\ ${ }^{1}$ School of Mechanical Engineering, Changzhou University, Changzhou 213016, China \\ ${ }^{2}$ School of Mechanical Engineering, Dalian University of Technology, Dalian 116024, China \\ ${ }^{3}$ Laboratoire des Sciences du Numérique de Nantes (LS2N), \\ UMR CNRS 6004, 1 rue de la Noe, 44321 Nantes, France \\ shp65@126.com,936515322@qq.com,gwu@dlut.edu.cn,wangju0209@163.com,Damien.Chablat@cnrs.fr
}

\begin{abstract}
This paper presents the kinematic design of a translational parallel mechanism (PM) named Vari-Orthoglide by means of the workspace superposition, according to the sub-kinematic chain (SKC) based PM composition principle. The main topological characteristics of the manipulator with two SKCs under study, such as the position and orientation (POC) characteristics, degree of freedom (DOF) and coupling degree are analyzed, which turns out that the coupling degree equals to 1 , implying the partially decoupled motion. With the topological characteristics based kinematic modeling principle, a symbolic model of the kinematics is established to derive its symbolic direct and inverse kinematic solutions. Based upon the direct kinematic solution, the workspaces for the two SKCs can be efficiently found. Moreover, the singularity loci are identified for finding the singularity-free workspace, where a regular workspace is fitted as the task workspace as expected. The presented work shows an approach to design translational parallel mechanisms considering motion decoupling and regular workspace, applicable to other types of parallel mechanisms.
\end{abstract}

Keywords: translational parallel mechanism, coupling degree, sub-kinematic chain, singularity, regular workspace

\section{Introduction}

Parallel mechanism (PM) based robotics and machines have found their applications in many fields, thanks to the advantages of low inertia, high speed, light weight, isotropic performances, simple inverse kinematics, etc. In the robotic applications, such as, machining and pick-and-place operations (PPOs), the family of three-degree-of-freedom (3-DOF) translational parallel mechanisms (TPMs) is one of the most commonly used robot counterparts, which means that TPMs have significant potentials of industrial application. One of the most popular is the classical parallel Delta robot $^{[1][2]}$, which has been successfully applied in the industrial PPOs in production lines ${ }^{[3]}$. Afterwards, a number of researchers reported different Delta mechanism variants by changing the type of actuated joints and the architectural layout of the chains ${ }^{[4]-[9]}$. Other types of translational parallel mechanisms have been extensively reported in the literature $^{[10]-[13]}$, too. For instance, a class of typical
TPMs consists of three $\mathrm{UPU}^{1}$ or PUU kinematic linkages ${ }^{[14]-[17]}$. Another approach to construct the TPMs is commonly to adopt the C-joint ${ }^{[18]}$ in the kinematic linkages, say 3-URC ${ }^{[19][20]}, 3-\mathrm{PRC}^{[21][22]}$, $3-\mathrm{CPU}^{[23]}$, etc. When the three linkages are arranged with a Cartesian configuration, i.e., the actuated joints located in three perpendicular planes, the TPMs can have the characteristics of completely decoupled motions and kinematic isotropy ${ }^{[24]-[26]}$. Moreover, the parallelogram ( $\mathrm{Pa}$, a.k.a, $\Pi$ joint) structure is an important linkage to lay out TPMs, thanks to the unique translational output motion, for example, the Orthoglide ${ }^{[27]}$ and among others ${ }^{[28]}$. Besides, a comparative study between the linearly and rotationally actuated TPMs have been carried out to show that the rotationally driven robots has better dynamic characteristics, while the ones with linear actuation has larger workspace ${ }^{[6]}$.

When the parallel mechanism is deployed in industrial application, the quality of the workspace

\footnotetext{
${ }^{1}$ Throughout this work, U, P, C and R represent universal, prismatic, cylindrical and revolute joints, respectively.
} 
that reflects the shape, size and presence of singularities is of primary importance in the PMs design ${ }^{[29]}$. Workspace based design can usually be solved with two different formulations, the first formulation aiming to design a manipulator whose workspace contains a prescribed workspace ${ }^{[30]}$ and the second approach being to design a manipulator whose workspace is as large as possible ${ }^{[31]}$. In practice, a task workspace with a regular geometry within the reachable workspace, such as sphere, cuboid, cylinder, etc., is an important measurement for the PM design ${ }^{[32]-[36]}$, whereas, the reachable workspace is generally irregularly shaped. Most of the exsiting TPMs have fully symmetrical topological architectures, which admits the existence of large regular workspace volume. On the other hand, the coupled input-output motion of those PMs leads to the highly nonlinear kinematic models, introducing the difficulties in the motion control and trajectory planning. Therefore, the design of new TPMs with the characteristics of decoupled kinematics is still one of the open questions.

In this work, a coupled mechanism means that the relationship between input and output motion variables of the mechanism are coupled, introducing the difficulties in the motion control and trajectory planning. A decoupled mechanism means that the relationship between input and output motion parameters of the mechanism are fully decoupled, which will ease the motion control and trajectory planning ${ }^{[37]}$. While, a partially decoupled mechanism means that the relationship between input and output motion parameters of the mechanism are partially decoupled, which also ease the motion control and trajectory planning compared to the fully coupled mechanisms. The asymmetric architecture can ensure advantages of motion decoupling for mechanisms, but usually results in the irregular workspace due to the asymmetry. Henceforth, the focus of this paper is to design the translational PMs with asymmetric structure and large regular workspace.

This paper combines the composition principle of PM based on sub-kinematic chain (SKC) ${ }^{[38]}$ and the superposition principle of sub-workspace determined by SKC units to design a 3-DOF TPM called Vari-Orthoglide, featuring asymmetric architecture with two SKCs as well as partially decoupled motions. The direct and inverse kinematic solutions of the PM were solved in a symbolic formulation. The corresponding workspace and singular configurations were analyzed to identify a cylindrical workspace free of singularities. The paper presents an approach to design translational parallel mechanisms and among others considering motion decoupling and regular workspace.

\section{Design method of parallel mechanisms based on POC and SKC}

\subsection{Basic theory of topological structure design based on position and orientation}

2.1.1 Position and orientation set (POC)

The POC equation of a $\mathrm{PM}^{[38]}$ is:

$$
\begin{aligned}
M_{b i} & =\bigvee_{i=1}^{m} M_{J i} \\
M_{P a} & =\coprod_{i=1}^{n} M_{b i}
\end{aligned}
$$

where

$M_{J i}$ - POC set generated by the $i^{\text {th }}$ joint.

$M_{b i}$ - POC set generated by the end link of $i^{\text {th }}$ branch chain.

$M_{P a}$ - POC set generated by the moving platform of PM.

\subsubsection{DOF calculation of parallel mechanism}

The DOF calculation of a parallel mechanism is mathematically expressed as:

$$
\begin{gathered}
F=\sum_{i=1}^{m} f_{i}-\sum_{j=1}^{v} \xi_{L_{j}} \\
\xi_{L j}=\operatorname{dim} \cdot\left\{\left(\mathbf{I}_{i=1}^{j} M_{b_{i}}\right) \mathrm{Y} M_{b_{(j+1)}}\right\}
\end{gathered}
$$

where

$F$ - DOF of PM.

$f_{i}$ - DOF of the $i^{\text {th }}$ joint.

$m$ - number of all joints of the PM.

$v$ - number of independent loops of the PM, and

$v=m-n+1$.

$n$ - number of links.

$\xi_{L_{j}}$ - number of independent equations of the $j^{\text {th }}$ loop.

$\mathrm{I}_{i=1}^{j} M_{b_{i}}-$ POC set generated by the sub-PM formed

by the former $j$ branches.

$M_{b(j+1)}$ - POC set generated by the end link of $j+1$ sub-chains.

2.1.3 Mechanism composition principle and 
coupling degree calculation based on SKC

According to the principle of mechanism composition based on single-opened-chains (SOC) units $^{[38]}$, any $\mathrm{PM}$ can be decomposed into a series of Assur kinematic chains (AKC), and an AKC with $v$ independent loops can be decomposed into $v$ SOC. The constraint degree of the $j^{\text {th }} \mathrm{SOC}, \Delta_{j}$, is defined by

$$
\Delta_{j}=\sum_{i=1}^{m_{j}} f_{i}-I_{j}-\xi_{L_{j}}=\left\{\begin{array}{l}
\Delta_{j}^{-}=-5,-4,-3,-2,-1 . \\
\Delta_{j}^{0}=0 \\
\Delta_{j}^{+}=+1,+2,+3, \Lambda
\end{array}\right.
$$

In Eq. (5), $m_{j}$ is the joint number of the $j^{\text {th }} \mathrm{SOC}_{\mathrm{j}} ; f_{i}$ is the degree of freedom of the $i^{\text {th }}$ joint (excluding local degrees of freedom); $I_{j}$ is the driving vice number of the $j^{\text {th }} \mathrm{SOC}_{j}$; The meaning of $\xi_{L_{j}}$ is the same to the previous description. For an AKC, it must satisfy with the following equation ${ }^{[38]}$ :

$$
\sum_{j=1}^{v} \Delta_{j}=0
$$

Sequentially, the coupling degree of $\mathrm{AKC}$ is defined by

$$
\kappa=\Delta_{j}^{+}=\left|\Delta_{j}^{-}\right|=\frac{1}{2} \min \left\{\sum_{j=1}^{v}\left|\Delta_{j}\right|\right\}
$$

where $\min \left\{\sum_{j=1}^{v}\left|\Delta_{j}\right|\right\}$ is decomposed into $v \operatorname{SOC}\left(\Delta_{j}\right)$, thus, there can be multiple composition schemes, and $\left(\sum\left|\Delta_{j}\right|\right)$ is the smallest.

\subsection{Design principle of mechanism based on SKC sub-workspace superposition}

For a fully symmetrical three translational PM like the Delta mechanism, the task workspace within the reachable workspace is often selected as a cylinder. However, for most asymmetric three translational PMs, the reachable workspace is usually in an irregular shape. In this work, a workspace-oriented approach is to be presented.

Chablat et al. applied a modular design idea to design a 3-DOF PM for machining, and selected a 2-PPa planar parallel mechanism with two translations and zero rotation (2T0R) as the sub-design module ${ }^{[39]}$. This module generates two translations in a plane, and then the end of the output member of the 2-PPa planar mechanism and the third branch chain are connected to the moving platform, in order that the moving platform obtains a rotation based on the two translations. Finally, a three-degree-of-freedom PM with two translations and one rotation output (2T1R) is designed. By integrating the previous approach of modular design, this paper proposes an approach of PM design based on the superposition of SKC-unit determined sub-workspace. In this approach, the final workspace is the common volume of all the sub-workspaces determine by each independent SKC, therefore, the PM to be designed can have not only expected workspace, but partially decoupled kinematics.

Inspired by this design approach, this paper presents a method to design a translational PM with a regular workspace in the shape of cuboid. The basic idea is to design a PM containing two SKCs to achieve three translations output. The output motion, that is, a planar PM as a sub-module (first SKC) generates a two-translational output motion to produce a rectangular cross-section workspace, sequentially, by adding a third branch (second SKC), the planar rectangle is extended through translation to form a cuboid as the task workspace.

\section{Topological design of TPM}

\subsection{Design of sub-parallel mechanism}

\subsubsection{Design source of sub-parallel mechanism}

Topological design ${ }^{[40]}$ of the mechanism structure is carried out in two stages, namely, type synthesis of a PMs and its topological analysis of the PM.

The planar parallel mechanism with output motions of two translations and zero rotation (2TOR) is firstly used as a kinematic module $\left(\mathrm{SKC}_{1}\right)$, to determine a rectangular workspace in the plane. The POC set of the output link of the planar PM is expressed as $\left[\begin{array}{l}t^{2} \\ r^{0}\end{array}\right]$.

The 2-PPa $\mathrm{PM}^{[39]}$, as displayed in Fig. 1, is only composed of P- and R-joints. The mechanism has a symmetrical structure, and the topological structure of the left and right branches is exactly the same. The active joints at points $P_{1}$ and $P_{2}$ move along the rails, and the end point $S$ is the output of the mechanism. It has a simple structure and various topological architectures, which is a perfect planar mechanism for generating two translational outputs.

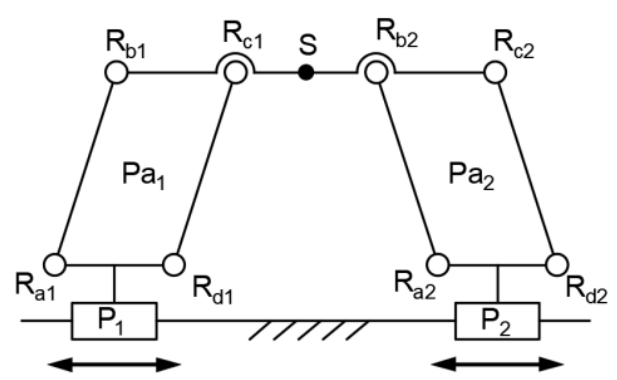

Fig.1 The diagram of 2-PPa planar parallel mechanism.

\subsubsection{Architectural simplification of 2-PPa PM}


Since the end of one single PPa branch in Fig. 1 already has two translation outputs, the other branch can be replaced by an unconstrained branch $P\|R\| R$. On the basis of Eqs. (2), (3), and (7), it can be proved that the main topological characteristics, e.g., the POC, DOF and coupling degree are same with the mechanism shown in Fig. 1. In this way, the structure of the mechanism is simplified as depicted in Fig. 2 to reduce the manufacturing cost.

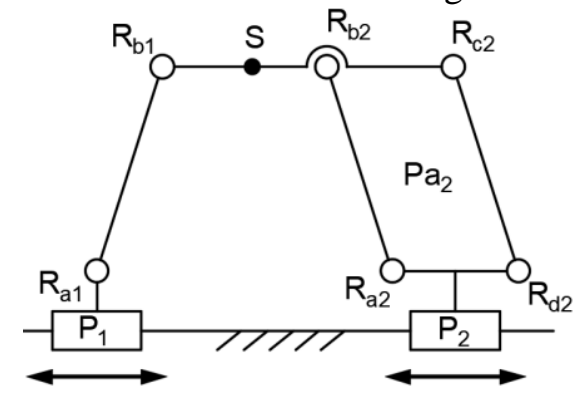

Fig.2 The alternative PRR-PPa planar parallel mechanism

(1) POC set analysis

The mechanism is made up of two single open chains connected in parallel, namely,

$$
\begin{gathered}
\mathrm{SOC}_{1}: \mathrm{P}_{1}-\mathrm{Ra}_{1}-\mathrm{R}_{\mathrm{b} 1} \\
\mathrm{SOC}_{2}: \mathrm{P}_{2}-\mathrm{Pa}_{2}
\end{gathered}
$$

According to Eq. (1), the POC sets of the output links for branch 1 and branch 2 are given by

$$
M_{b 1}=\left[\begin{array}{c}
t^{2} \\
r^{1}\left(\| \mathrm{R}_{b 1}\right)
\end{array}\right], \quad M_{b 2}=\left[\begin{array}{c}
t^{2} \\
r^{0}
\end{array}\right] .
$$

From Eq. (2), the POC set of the moving platform is derived as

$$
M_{p}=\left[\begin{array}{c}
t^{2}\left(\| \diamond a_{2} b_{2} c_{2} d_{2}\right) \\
r^{0}
\end{array}\right]
$$

(2) DOF analysis

The mechanism contains only one independent circuit, namely

$$
\operatorname{LOOP}_{2}\left\{-\mathrm{P}_{1}-\mathrm{R}_{\mathrm{a} 1}-\mathrm{R}_{\mathrm{b} 2}-\mathrm{P}_{\mathrm{a} 2}-\mathrm{P}_{2}-\right\}
$$

from which it is known that the number of independent displacement equations of the plane mechanism is $\xi_{L}=3$. Thus, the DOF of the PM is calculated as

$$
F=\sum_{i=1}^{m} f_{i}-\sum_{j=1}^{v} \xi_{L_{j}}=5-3=2
$$

(3) Coupling degree analysis

It can be obtained from Eq. (5) that the constraint degree of this loop is

$$
\Delta=\sum_{i=1}^{m_{1}} f_{i}-I_{1}-\xi_{L 1}=5-2-3=0
$$

subsequently, from Eq. (7), the coupling degree of the parallel mechanism is $\kappa=\frac{1}{2}|0|=0$. Therefore, the architectural simplified PM mechanism is selected as the sub-module mechanism $\left(\mathrm{SKC}_{1}\right)$ which can provide two translation output motions.

In Ref. [39], the 2(P\|R\|R) planar mechanism is used for machining, with the analysis of the effect of the arrangement of the prismatic joints onto the robot performance. The conclusions were drawn as that the workspace volume of the end-effector of the mechanism is constant, when the two $\mathrm{P}$ joints are arranged with perpendicular sliding axes, leading to the mechanism with a compact structure, a small inertia and a high payload capacity simultaneously. Accordingly, the modified planar PM is designed with perpendicular arrangement of the active joints, as shown in Fig. 3.

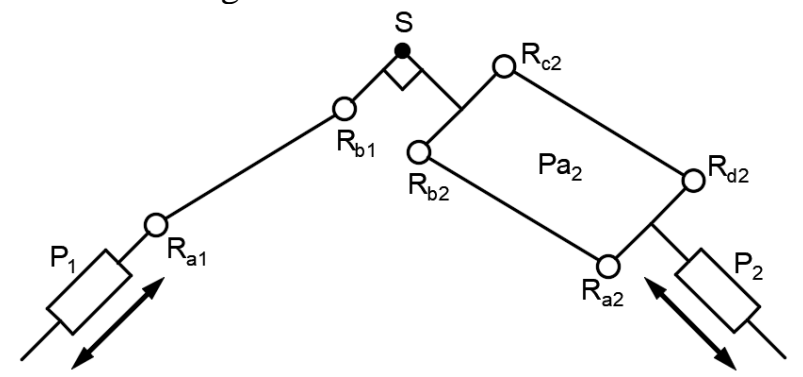

Fig.3 Modified PRR-PPa planar parallel mechanism

\subsection{Design of the overall translational PM}

According to the PM POC Eq. (2), the POC set of the moving platform of the mechanism is obtained by the "intersection" operation of the POC set at the end of each branch chain; and the algorithm of the "intersection" operation ${ }^{[38]}$ makes the end moving platform have three translational motion outputs, thus, the output link of each branch chain connected to the moving platform must have three-translation motion. Therefore, adding more kinematic joints in series at the ends of the two translational planar mechanisms can form a branch chain with three translational output motions.

In order to increase the translational output of the end, a $4 \mathrm{R}$ parallelogram mechanism is connected in series at the end, and its POC set is $\left[\begin{array}{c}t^{1}(\| \diamond a b c d) \\ r^{0}\end{array}\right]$; It can be seen from the POC Eq. (1) of the serial mechanism that the POC set of the end point $S$ of the new sub-parallel mechanism formed with the two mechanisms connected in series is written as $\left[\begin{array}{l}t^{3} \\ r^{0}\end{array}\right]$; So far, the first complex chain (Complex Chain, CC) has been designed, as shown in Fig. 4, noted as branch chain (a).

For the second complex branch, we use the $\{\mathrm{P}\|\mathrm{R} \perp(\mathrm{Pa})\| \mathrm{R}\}$ branch (b) in Fig. 4, the POC sets of the output link contain three translations and one 
rotation (3T1R). From Eq. (2), the POC set of the moving platform is

$$
M_{P a}=M_{C C 1} \mathrm{I} \quad M_{C C 2}=\left[\begin{array}{c}
t^{3} \\
r^{0}
\end{array}\right] \mathrm{I}\left[\begin{array}{c}
t^{3} \\
r^{1}(\| \mathrm{R})
\end{array}\right]=\left[\begin{array}{c}
t^{3} \\
r^{0}
\end{array}\right]
$$

Consequently, the expected three translational output motions of the moving platform is realized.

Since this mechanism is similar to the Orthoglide parallel mechanism ${ }^{[27]}$, it can be regarded as a modified variant, named as Vari-Orthoglide, for which the CAD model is displayed in Fig. 5.

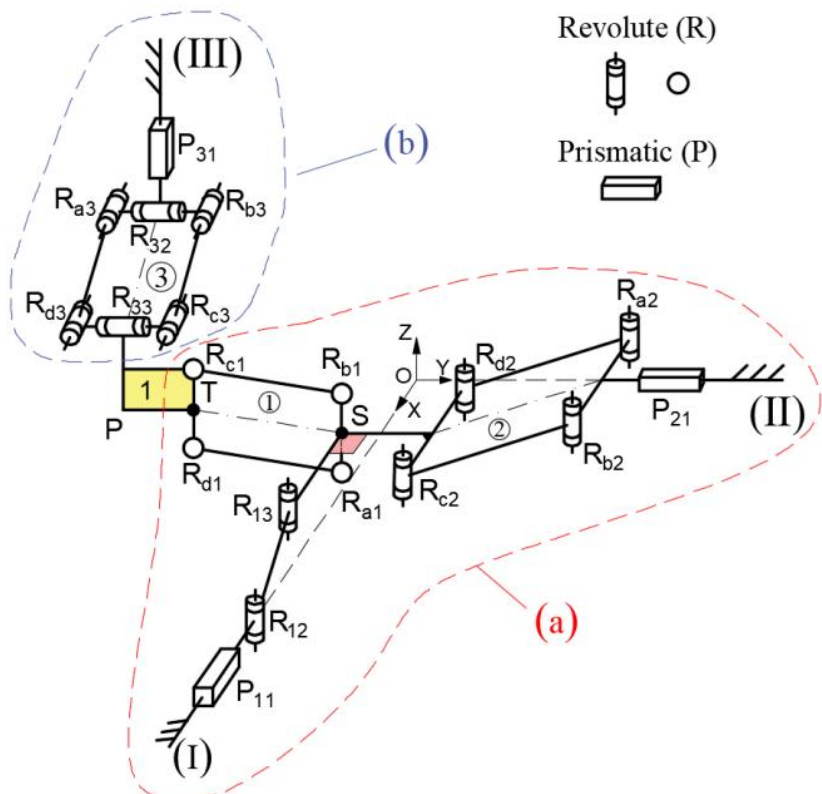

Fig.4 Schematic diagram of Vari-Orthoglide PM

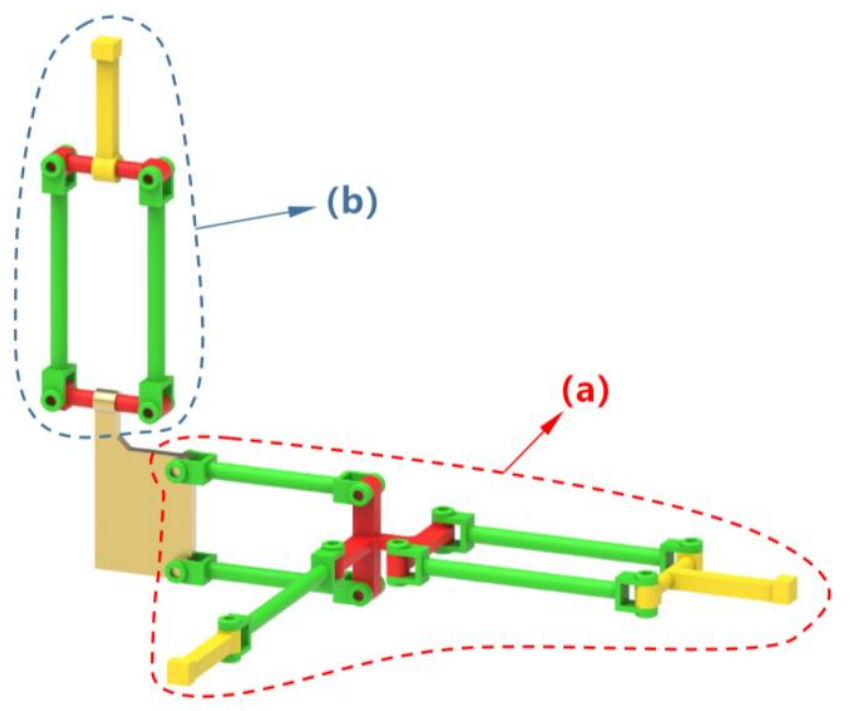

Fig.5 CAD model of Vari-Orthoglide PM

The topological architecture of the proposed Vari-Orthoglide PM, as depicted in Figs. 4 and 5, is described below:

- The PM consists of two complex chains
(Complex Chain, CC), namely (a) and (b) branches, which are connected together at both ends of the moving platform 1;

- The branch chain (a) is composed of the sub-parallel mechanism designed in Sect. 2.1.2 at the end point $S$, and a parallelogram mechanism (1) which is perpendicular to the motion plane of the sub-parallel mechanism is connected in series to form the first hybrid Branch 1 (denoted as $\mathrm{CC}_{1}$ ).

- The branch chain (b) consists of a prismatic joint $\mathrm{P}_{31}$ and a $4 \mathrm{R}$ parallelogram mechanism connected in series through a $\mathrm{R}$-joint $\mathrm{R}_{32}$, and the end of the $4 \mathrm{R}$ parallelogram mechanism is connected to the moving platform 1 through the $\mathrm{R}$-joint $\mathrm{R}_{33}$ to form a complex branch chain 2 (denoted as $\mathrm{CC}_{2}$ ).

- The active P-joints $\mathrm{P}_{11}$ and $\mathrm{P}_{21}$ are located in the same plane $O-X Y$, with the moving axes parallel to $X$ - and $Y$-axis, respectively, while the active P-joint $\mathrm{P}_{31}$ moves along $Z$-axis, yielding a Cartesian parallel mechanism.

Henceforth, the proposed Vari-Orthoglide PM has the following advantages:

- All the joints are revolute pairs except the actuated ones to ease the fabrication;

- The mechanism has simple analytical solutions of direct and inverse kinematic positions that is beneficial to dynamic analysis, trajectory planning and control;

- The motion of the moving platform along $Y$-axis is determined by active joints $\mathrm{P}_{11}$ and $\mathrm{P}_{21}$, due to its partially decoupled kinematics, to ease the control;

- Each SKC module can be separately optimized or improved upon the design or application requirements. Amongst the previous advantages, the Orthoglide also has the first two aspects.

\subsection{Topological analysis of Vari-Orthoglide PM 3.3.1 POC set analysis}

It is known from Eq. (2) that the POC set of the moving platform of the mechanism is obtained by the "intersection" operation from the POC sets of the two complex branches $\mathrm{CC}_{1}$ and $\mathrm{CC}_{2}$. Thus, the POC set of each branch is to be determined firstly.

According to Eq. (1), the POC set of the output link in a single kinematic chain is deduced by the "union" operation, as a consequence, the POC sets of two complex branches CC are analyzed below

(1) Select the position of point $\mathrm{P}$ on moving platform 1 as the reference point $\mathrm{O}^{\prime}$;

(2) Determine the POC set of the output link in branch $\mathrm{CC}_{1}$. The topological architecture of branch 
$\mathrm{CC}_{1}$ is depicted as

$$
\mathrm{CC}_{1}\left\{-\diamond\left(\mathrm{P}_{11} \perp \mathrm{R}_{12}\left\|\mathrm{R}_{13}-\mathrm{Pa}_{(2)}\right\| \mathrm{P}_{21}\right) \perp \mathrm{Pa}_{(1)}^{(4 \mathrm{R})}-\right\}
$$

Let the POC sets of the output link in the sub-parallel mechanism and $4 \mathrm{R}$ parallelogram mechanism be denoted by $\mathrm{M}_{\text {sub }}$ and $\mathrm{M}_{2}$, respectively, one obtains

$$
\begin{gathered}
M_{\text {sub }}=\left[\begin{array}{c}
t^{2}\left(\| \diamond a_{2} b_{2} c_{2} d_{2}\right) \\
r^{0}
\end{array}\right] \\
M_{2}=\left[\begin{array}{c}
t^{1}\left(\| \diamond\left(\mathrm{a}_{1} \mathrm{~b}_{1} \mathrm{c}_{1} \mathrm{~d}_{1}\right)\right) \\
r^{0}
\end{array}\right]
\end{gathered}
$$

According to Eq. (1), the POC set of the output link in branch 1 can be expressed as

$$
M_{C C 1}=\left[\begin{array}{c}
t^{2}\left(\| \diamond a_{2} b_{2} c_{2} d_{2}\right) \\
r^{0}
\end{array}\right] \mathrm{Y}\left[\begin{array}{c}
t^{1}\left(\| \diamond\left(\mathrm{a}_{1} \mathrm{~b}_{1} \mathrm{c}_{1} \mathrm{~d}_{1}\right)\right) \\
r^{0}
\end{array}\right]=\left[\begin{array}{l}
t^{3} \\
r^{0}
\end{array}\right]
$$

(3) Determine the POC set of the output link in the branch $\mathrm{CC}_{2}$. The topological architecture of branch $\mathrm{CC}_{1}$ is depicted as

$$
\mathrm{CC}_{2}\left\{-\mathrm{P}_{31}\left\|\mathrm{R}_{32}\left(\perp \mathrm{Pa}^{(4 \mathrm{R})}\right)\right\| \mathrm{R}_{33}-\right\}
$$

Hence, the POC set is derived as

$$
M_{C C 2}=
$$

$\left[\begin{array}{c}t^{1}\left(\| \mathrm{P}_{31}\right) \\ r^{0}\end{array}\right] \mathrm{Y}\left[\begin{array}{c}t^{1}\left(\perp \mathrm{R}_{32}\right) \\ r^{1}\left(\| \mathrm{R}_{32}\right)\end{array}\right] \mathrm{Y}\left[\begin{array}{c}t^{1}\left(\| \diamond\left(a_{2} b_{2} c_{2} d_{2}\right)\right) \\ r^{0}\end{array}\right] \mathrm{Y}\left[\begin{array}{c}t^{1}\left(\perp \mathrm{R}_{33}\right) \\ r^{1}\left(\| \mathrm{R}_{33}\right)\end{array}\right]$ $=\left[\begin{array}{c}t^{3} \\ r^{1}\left(\| \mathrm{R}_{33}\right)\end{array}\right]$

(4) Determine the POC set of the moving platform. Based upon Eq. (2), the POC set of the moving platform is derived from the intersection of the two branches, namely,

$$
M_{P a}=M_{C C 1} \mathrm{I} M_{C C 2}=\left[\begin{array}{l}
t^{3} \\
r^{0}
\end{array}\right] \mathrm{I}\left[\begin{array}{c}
t^{3} \\
r^{1}\left(\| \mathrm{R}_{33}\right)
\end{array}\right]=\left[\begin{array}{c}
t^{3} \\
r^{0}
\end{array}\right]
$$

which turns out that the output motion of the moving platform of the mechanism is a spatial translation.

\subsubsection{DOF analysis of the PM}

The mechanism can be decomposed into two independent loops connected in serial, thus, the first loop is the sub-parallel mechanism $\left(\mathrm{SKC}_{1}\right)$ in branch 1 , namely,

$$
\text { LOOP }\left\{-\mathrm{P}_{11} \perp \mathrm{R}_{12}\left\|\mathrm{R}_{13}-\mathrm{Pa}_{2}\right\| \mathrm{P}_{21}-\right\}
$$

and the second one is composed of the former sub-parallel mechanism and a sub-chain

$$
\operatorname{SOC}\left\{-\mathrm{Pa}_{(1)}^{(4 \mathrm{R})}-\mathrm{R}_{33}\left(\perp \mathrm{Pa}_{(3)}^{(4 \mathrm{R})}\right)\left\|\mathrm{R}_{32}\right\| \mathrm{P}_{31}-\right\} \text {. }
$$

(1) Determine the number of independent displacement equations of the first loop

From Eq. (8), the number of independent displacement equations of the first loop is $\xi_{L_{1}}=3$, therefore, the DOF from Eq. (3) is calculated as

$$
F_{2}=\sum_{i=1}^{m} f_{i}-\sum_{j=1}^{v} \xi_{L_{j}}=5-3=2
$$

which means that the output motion of the sub-parallel mechanism is a 2-DOF translations.

(2) Determine the number of independent displacement equations of the second loop

From Eq. (4), we have

$$
\begin{aligned}
\xi_{L_{2}} & =\operatorname{dim} .\left\{M_{\text {sub }} \mathrm{Y} M_{C C 2} \mathrm{Y} M_{2}\right\} \\
& =\operatorname{dim} .\left\{\left[\begin{array}{c}
t^{3} \\
r^{1}\left(\| R_{33}\right)
\end{array}\right]\right\}=4
\end{aligned}
$$

(3) Determine the mechanism DOF

From eq. (3), the DOF of the mechanism is

$$
F=\sum_{i=1}^{m} f_{i}-\sum_{j=1}^{v} \xi_{L_{j}}=10-(3+4)=3
$$

To this end, the DOF of the mechanism is equal to 3 , when the $\mathrm{P}$-joints $\mathrm{P}_{11}, \mathrm{P}_{21}$ and $\mathrm{P}_{31}$ on the fixed platform 0 are selected as the driving joints, of which the output motions of the moving platform are spatial translations.

3.3.3 Analysis of coupling degree

The number of independent displacement equations of the two loops have been calculated in the previous section, respectively, namely, $\xi_{L 1}=3$, $\xi_{L 2}=4$. Accordingly, the constraint degree of the two loops from Eq. (5) are calculated as

$$
\begin{aligned}
& \Delta_{1}=\sum_{i=1}^{m_{1}} f_{i}-I_{1}-\xi_{L 1}=5-2-3=0 \\
& \Delta_{2}=\sum_{i=1}^{m_{2}} f_{i}-I_{2}-\xi_{L 2}=5-1-4=0
\end{aligned}
$$

Consequently, in accordance with Eq. (6), loops 1 and 2 consisting of first and second SKCs, that is, the mechanism is composed of two SKCs with zero constraint degree. Now from Eq. (7), the coupling degrees of the two loops are both equal to 0 , which yields

$$
\kappa=\frac{1}{2} \sum_{j=1}^{v}\left|\Delta_{j}\right|=\frac{1}{2}(|0|)=0
$$

Finally, it is verified that the coupling degree of the proposed Vari-Orthoglide PM is $\kappa=0$.

\section{Kinematic analysis of Vari-Orthoglide PM}

4.1 Parametric modeling of mechanism As the Vari-Orthoglide PM is designed based on the superposition principle of the SKC unit sub-workspace, its kinematic analysis can be accomplished with the single SKC unit. For convenience, the schematic diagram of the mechanism in Fig. 4 is reformed, as shown in Fig. 6. 


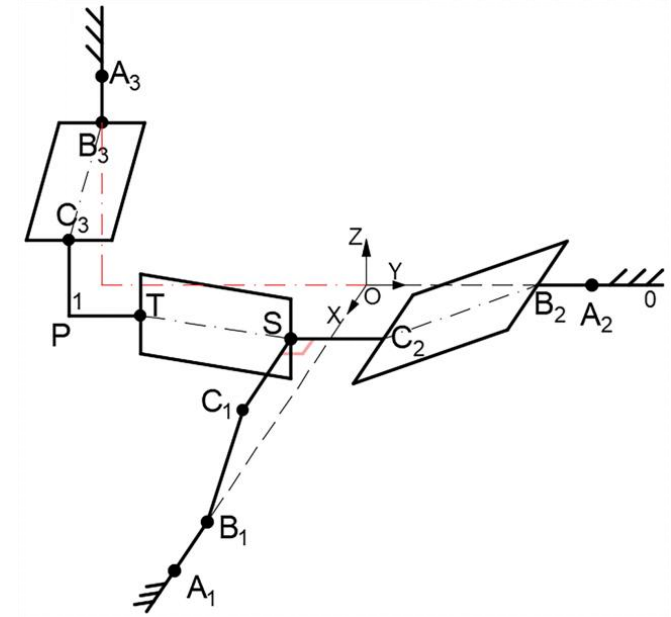

Fig.6 Simplified Vari-Orthoglide PM schematic diagram

For a better understanding, two side views for the branches chain $\mathrm{CC}_{1}$ and $\mathrm{CC}_{2}$ are shown in Fig. 7 and 8, respectively. In Figs. 7 and 8, point $A_{i}, i=1 \sim 3$, represent the position of the three active $\mathrm{P}$-joints $\mathrm{P}_{11}$, $\mathrm{P}_{21}$ and $\mathrm{P}_{31} ; B_{1}$ and $B_{3}$ represent the positions of the $\mathrm{R}$-joints $\mathrm{R}_{12}$ and $\mathrm{R}_{32}$, and $B_{2}$ represents the midpoint of the connecting rod in the $4 \mathrm{R}$ parallelogram.

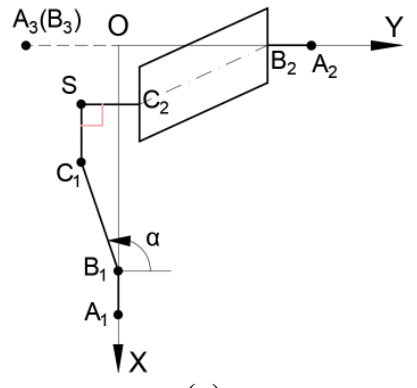

(a)

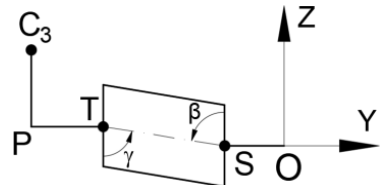

(b)
Fig.7 Side views of branch $\mathrm{CC}_{1}$ : (a) projection in plane $X O Y$; (b) projection in plane $Y O Z$

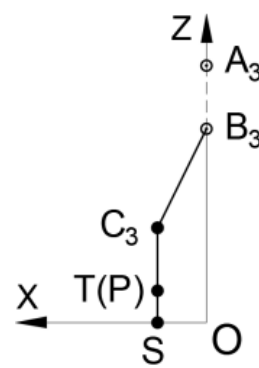

Fig.8 Side view of branch $\mathrm{CC}_{2}$ in $\mathrm{XOZ}$ plane

The reference coordinate frame $(X, Y, Z)$ is built with the origin located at the intersection point of moving axes of active $\mathrm{P}$-joints $\mathrm{P}_{11}$ and $\mathrm{P}_{21}$, where $X$ - and $Y$-axes are parallel to the moving axes. The moving coordinate frame $(x, y, z)$ is built with the origin located at point $P$, whose axes are parallel to those of the reference frame. Moreover, in branch 1, the link lengths are parameterized as $A_{1} B_{1}=l_{11}$, $A_{2} B_{2}=l_{21}, \quad B_{1} C_{1}=l_{12}, \quad B_{2} C_{2}=l_{22}, \quad C_{1} S=l_{13}, \quad C_{2} S=l_{23}$, $S T=l_{4}, T P=l_{5}$, and in branch $2, A_{3} B_{3}=l_{31}, B_{3} C_{3}=l_{8}$, and $C_{3} P=l_{6}$.

\subsection{Forward and inverse kinematic analysis}

\subsubsection{Forward position analysis}

Let suppose the joint variables of the three active P-joints to be cast in a vector $\rho=\left(\rho_{1}, \rho_{2}, \rho_{3}\right)$, the objective of forward position analysis is to find the position vector of reference point $P=\left[P_{x}, P_{y}, P_{z}\right]^{T}$ on the moving platform with prescribed input joint variables.

From Fig. 6, the position vectors of the active $\mathrm{P}$-joints in the reference frame are expressed as

$$
A_{1}=\left[\rho_{1}, 0,0\right]^{T}, A_{2}=\left[0, \rho_{2}, 0\right]^{T}, A_{3}=\left[0,-l_{7}, \rho_{3}\right]^{T}
$$

and the position vectors of points $B_{1}, B_{2}$ and $B_{3}$ can be derived as.

$$
\begin{aligned}
& B_{1}=\left[\rho_{1}-l_{11}, 0,0\right]^{T} \\
& B_{2}=\left[0, \rho_{2}-l_{21}, 0\right]^{T} \\
& B_{3}=\left[0,-l_{7}, \rho_{3}-l_{31}\right]^{T}
\end{aligned}
$$

It is known from Section 3.3.3 that the mechanism is composed of two $\mathrm{SKC}_{1}$ and $\mathrm{SKC}_{2}$ with zero constraint degree. With the method to solve the forward position problem ${ }^{[44]}$ based on the topological characteristics ${ }^{[41]}$, displacement analysis is carried out along with the two independent SKCs.

(1) Displacement solution of $\mathrm{SKC}_{1}$

To find the forward displacement of the first loop $\left(\mathrm{A}_{1}-\mathrm{B}_{1}-\mathrm{C}_{1}-\mathrm{S}-\mathrm{C}_{2}-\mathrm{B}_{2}-\mathrm{A}_{2}\right)$, namely,

$$
\text { LOOP }\left\{-\mathrm{P}_{11}-\mathrm{R}_{12}-\mathrm{R}_{13}-\mathrm{S}-\mathrm{P}_{(2)}^{(4 \mathrm{R})}-\mathrm{P}_{21}-\right\}
$$

Thus, the Cartesian coordinates of points $C_{1}, S$ and $C_{2}$ can be expressed in terms of Cartesian coordinates of point $B_{1}$ below

$$
\begin{gathered}
C_{1}=\left[x_{B 1}-l_{12} \sin \alpha, y_{B 1}+l_{12} \cos \alpha, 0\right]^{T} \\
S=\left[x_{C 1}-l_{13}, y_{C 1}, 0\right]^{T} \\
C_{2}=\left[x_{S}, y_{S}+l_{23}, 0\right]^{T}
\end{gathered}
$$

By making use of the geometric constraint $C_{2} B_{2}=l_{2}$, the loop-closure equation is written as

$$
F_{1} \sin \alpha+F_{2} \cos \alpha+F_{3}=0
$$

where

$$
\begin{gathered}
F_{1}=-2 D l_{12}, \quad D=\rho_{1}-l_{11}-l_{13} \\
F_{2}=2 E l_{12}, \quad E=l_{23}-\rho_{2}+l_{21} \\
F_{3}=D_{2}+E_{2}+l_{12}^{2}-l_{22}^{2}
\end{gathered}
$$

Let $\tan \frac{\alpha}{2}=\mu$, one obtains 


$$
\alpha=2 \arctan \frac{-F_{1}+m \sqrt{F_{1}^{2}+F_{2}^{2}-F_{3}^{2}}}{F_{3}-F_{2}},(m= \pm 1)
$$

(2) Displacement solution of $\mathrm{SKC}_{2}$

Similarly, to solve the forward position of loop $2\left(\mathrm{~S}-\mathrm{T}-\mathrm{P}-\mathrm{C}_{2}-\mathrm{B}_{3}-\mathrm{A}_{3}\right)$ below

$$
\mathrm{SOC}\left\{-\mathrm{P}_{31}\left\|\mathrm{R}_{32}\left(\perp \mathrm{Pa}_{(3)}^{(4 \mathrm{R})}\right)\right\| \mathrm{R}_{33}-P-\mathrm{Pa}_{(1)}^{(4 \mathrm{R})}-\right\}
$$

The Cartesian coordinates of point $T$ can be expressed in terms of Cartesian coordinates of point $\mathrm{S}$ as below

$$
T=\left[x_{S}, y_{S}-l_{4} \sin \beta, z_{S}+l_{4} \cos \beta\right]^{T}
$$

Moreover, the Cartesian coordinates of point $P$ can be expressed with those of point $T$.

After the matrix operation, the forward position of the reference point $P$ on the moving platform is solved as

$$
P=\left[\begin{array}{c}
x_{T} \\
y_{T}-l_{5} \\
z_{T}
\end{array}\right]=\left[\begin{array}{c}
\rho_{1}-l_{11}-l_{12} \sin \alpha-l_{13} \\
l_{12} \cos \alpha+l_{4} \sin \beta-l_{5} \\
l_{4} \cos \beta
\end{array}\right]
$$

Now the forward kinematic solution in Eq. (12) still contains an unknown variable $\beta$, thus, one more constraint equation from $C_{3} B_{3}=l_{8}$ is added

$$
K_{1} \sin \beta+K_{2} \cos \beta+K_{3}=0
$$

with

$$
\begin{gathered}
K_{1}=-2 H l_{4} \\
K_{2}=2 J l_{4} \\
K_{3}=G^{2}+H^{2}+J^{2}+l_{4}^{2}-l_{8}^{2}
\end{gathered}
$$

where

$$
\begin{gathered}
G=\rho_{1}-l_{11}-l_{12} \sin \alpha-l_{13} \\
H=l_{12} \cos \alpha-l_{5}+l_{7} \\
J=l_{6}-\rho_{3}+l_{31}
\end{gathered}
$$

Let $\tan \frac{\beta}{2}=t$, variable $\beta$ can be solved as

$$
\beta=2 \arctan \frac{-K_{1}+n \sqrt{K_{1}^{2}+K_{2}^{2}-K_{3}^{2}}}{K_{3}-K_{2}} n= \pm 1
$$

Substituting Eqs. (11) and (13) into Eq. (12), the Cartesian coordinates of the reference point $\mathrm{P}$ on the moving platform can be obtained, which are the functions of the input motion variables as below

$$
\left\{\begin{array}{l}
P_{x}=f_{1}\left(\rho_{1}, \rho_{2}, \rho_{3}\right) \\
P_{y}=f_{2}\left(\rho_{1}, \rho_{2}\right) \\
P_{z}=f_{3}\left(\rho_{1}, \rho_{2}, \rho_{3}\right)
\end{array}\right.
$$

From the forward position analysis, it is found that the manipulator can have four solutions, corresponding to four assembly modes. Moreover, the $Y$-coordinate of point $P$ is determined by the first two active P-joints, which corresponds to its partially decoupled motion.

\subsubsection{Inverse position analysis}

The inverse kinematics of the PM is to find the joint variables $\rho=\left(\rho_{1}, \rho_{2}, \rho_{3}\right)$ with prescribed position vector $P=\left[P_{x}, P_{y}, P_{z}\right]^{T}$ of the reference point $P$ on the moving platform

Given the Cartesian coordinates of point $P$, the position vector of points $T$ and $S$ can be respectively expressed as

$$
\begin{gathered}
T=\left[x_{P}, y_{P}+l_{5}, z_{P}\right]^{T} \\
S=\left[x_{T}, y_{T}+l_{4} \sin \gamma, z_{T}-l_{4} \cos \gamma\right]^{T}
\end{gathered}
$$

yielding the position vectors of points $C_{1}$ and $C_{2}$

$C_{1}=\left[x_{S}+l_{13}, y_{S}, z_{S}\right]^{T}, C_{2}=\left[x_{S}, y_{S}+l_{23}, z_{S}\right]^{T}$

Since the $Z$-coordinate of points $C_{1}$ and $C_{2}$ are both equal to zero, namely,

$$
z_{C 1}=z_{C 2}=P_{z}-l_{4} \cos \gamma=0
$$

thus, one obtains

$$
\cos \gamma=\frac{P_{z}}{l_{4}}, \quad \sin \gamma= \pm \sqrt{1-\left(\frac{P_{z}}{l_{4}}\right)^{2}}
$$

By the same token, the position vector of point $C_{3}$ can be derived from the coordinates of point $\mathrm{P}$ as

$$
C_{3}=\left[x_{P}, y_{P}, z_{P}+l_{6}\right]^{T}
$$

From the geometric constraints $B_{1} C_{1}=l_{12}, B_{2} C_{2}=l_{22}$ and $B_{3} C_{3}=l_{8}$, the kinematic constraint equations are obtained as

$$
\left\{\begin{array}{l}
\left(x_{B 1}-x_{C 1}\right)^{2}+\left(y_{B 1}-y_{C 1}\right)^{2}+\left(z_{B 1}-z_{C 1}\right)^{2}-l_{12}^{2}=0 \\
\left(x_{B 2}-x_{C 2}\right)^{2}+\left(y_{B 2}-y_{C 2}\right)^{2}+\left(z_{B 2}-z_{C 2}\right)^{2}-l_{22}^{2}=0 \\
\left(x_{B 3}-x_{C 3}\right)^{2}+\left(y_{B 3}-y_{C 3}\right)^{2}+\left(z_{B 3}-z_{C 3}\right)^{2}-l_{8}^{2}=0
\end{array}\right.
$$

which can be simplified as

$$
\left\{\begin{array}{l}
\left(\rho_{1}+M_{1}\right)^{2}+N_{1}=0 \\
N_{2}+\left(\rho_{2}+M_{2}\right)^{2}=0 \\
N_{3}+\left(\rho_{3}+M_{3}\right)^{2}=0
\end{array}\right.
$$

where,

$$
M_{1}=-l_{11}-\left(P_{x}+l_{13}\right) ; N_{1}=\left(P_{y}+l_{5}+l_{4} \sin \gamma\right)^{2}-l_{12}^{2}
$$

$$
\begin{gathered}
M_{2}=-l_{21}-\left(P_{y}+l_{5}+l_{4} \sin \gamma+l_{23}\right) ; N_{2}=P_{x}^{2}-l_{22}^{2} \\
M_{3}=-l_{31}-\left(P_{z}+l_{6}\right) ; N_{3}=P_{x}^{2}+\left(l_{7}+P_{y}\right)^{2}-l_{8}^{2}
\end{gathered}
$$

Solving Eq. (20) results in

$$
\rho_{i}=-M_{i}+S_{j} \sqrt{-N_{i}} \quad\left(i=1,2,3 ; j=x, y, z, S_{j}= \pm 1\right)
$$

From Eqs. (17) and (21), it is seen that the 
mechanism can have up to 16 solutions to the inverse position analysis, which associates with 16 working modes.

\subsubsection{Numerical verification of kinematic model}

Let the geometric parameters (in the unit of $\mathrm{mm}$ ) of the Vari-Orthoglide PM be set to

$$
\begin{aligned}
& l_{11}=l_{21}=l_{31}=100, l_{12}=l_{22}=230, l_{13}=75, l_{23}=50, \\
& l_{4}=170, l_{5}=70, l_{6}=95, l_{7}=240, l_{8}=210
\end{aligned}
$$

(1) Example of forward position analysis

With the prescribed parameters, a CAD model is built with SolidWorks ${ }^{\mathrm{TM}}$, and two sets of arbitrary input joint variables and positions of the moving platform are measured listed in Table 1 to evaluate the analytical model.

Table 1 Two measured configurations of CAD model

\begin{tabular}{cll}
\hline Configurations & Joint variables & MP position \\
\hline \multirow{2}{*}{1} & $\rho_{1}=296.96$ & $x=-52.84$ \\
& $\rho_{2}=353.39$ & $y=-203.94$ \\
& $\rho_{3}=247.01$ & $z=-87.84$ \\
\hline \multirow{2}{*}{2} & $\rho_{1}=287.14$ & $x=-61.38$ \\
& $\rho_{2}=315.63$ & $y=-246.37$ \\
& $\rho_{3}=261.28$ & $z=-73.18$ \\
\hline
\end{tabular}

The analytical solutions of the moving platform positions corresponding to measured joint variables in Table 1 are given in Table 2. It is seen that when the sign $\mathrm{m}$ in Eq. (11) is negative, angle $\beta$ does not exist. With the working mode $m=+1$ and $n=+1$ for the mechanism under study, the analytical solutions have a good agreement with the measured ones, which shows the validation of the analytical model for the forward position analysis.

Table 2 Analytical solutions of the forward position

\begin{tabular}{c|c|c|c|c|c|c}
\hline Set & $m$ & $n$ & No. & $x$ & $y$ & $z$ \\
\hline \multirow{4}{*}{$(1)$} & +1 & +1 & $1^{*}$ & -52.8420 & -203.9380 & -87.8313 \\
\cline { 2 - 7 } & +1 & -1 & 2 & -52.8420 & -78.0593 & 185.1623 \\
\cline { 2 - 7 } & -1 & +1 & 3 & - & - & - \\
\cline { 2 - 7 } & -1 & -1 & 4 & - & - & - \\
\hline \multirow{4}{*}{$(2)$} & +1 & +1 & $1 *$ & -61.3825 & -246.3730 & -73.1715 \\
\cline { 2 - 7 } & +1 & -1 & 2 & -61.3825 & -75.8355 & 189.9392 \\
\cline { 2 - 7 } & -1 & +1 & 3 & - & & - \\
\cline { 2 - 7 } & -1 & -1 & 4 & - & & - \\
\hline
\end{tabular}

(2) Example of inverse position analysis

As can be seen from Figs 6 and 7, the $Y$-coordinate of point $S$ for a real mechanism must be greater than the $Y$-coordinate of point $T$ to ensure the avoidance of mechanical collision. Therefore, the angle $\gamma$ can only be in the range $[0, \pi / 2]$, which means that $\cos \gamma$ is always nonnegative, leading to the vanished 8 sets of solutions of the inverse position from $\mathrm{Eq}$ (17). Moreover, amongst the 8 remaining sets of solutions corresponding to the assembly modes, only one configuration is feasible.

In order to interpret the different assembly modes, i.e., solutions of inverse geometry, Fig. 9 shows different configurations when the reference point $P$ is located at an arbitrary position. When the active $\mathrm{P}$-joint is located in the positive coordinates in the reference frame $(X, Y, Z)$, "P" denotes the configuration, conversely, " $\mathrm{M}$ " for negative coordinates. For instance, when the three active P-joints are located in the positive coordinate axes, namely, $\left\{S_{x}, S_{y}, S_{z}\right\}=+1$ in Eq. (21), such as the configuration depicted by PPP as shown in Fig. 9(a). Figures 9(b) to 9(d) depicts the configurations PMP, PPM and MMP, respectively. In these four configurations, only the PPP candidate is feasible.

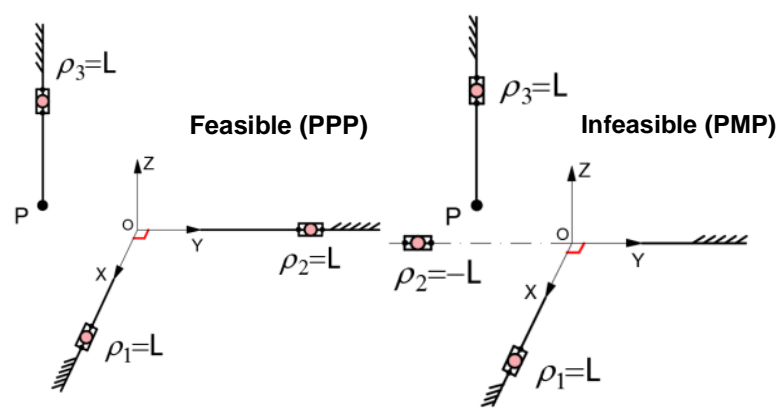

(a)

(b)

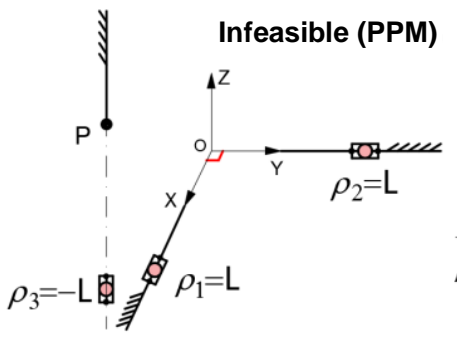

(c)

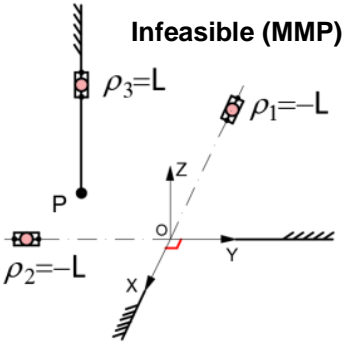

(d)
Fig.9 Feasible and non-feasible configurations: (a) PPP; (b) PMP; (c) PPM; (d) MMP

With the first moving platform position shown in Table 1, the analytical solutions for the inverse position analysis is solved in Table 3, which agrees well with the measure joint variables. After multiple verification of the forward and inverse kinematic models, the most feasible solutions to Eqs. (11) and (21) for the mechanism configuration are selected as:

$$
m=+1, n=+1, S_{x}=S_{y}=S_{z}=+1
$$

Table 3 Analytical solutions of the inverse position

\begin{tabular}{l|l|l|l|l}
\hline No. & Type & $\rho_{1}$ & $\rho_{2}$ & $\rho_{3}$ \\
\hline
\end{tabular}




\begin{tabular}{c|c|c|c|c}
\hline $1^{*}$ & PPP & 296.9622 & 353.3839 & 247.0022 \\
\hline 2 & PPM & 296.9622 & 353.3839 & -112.6822 \\
\hline 3 & PMP & 296.9622 & -94.3121 & 247.0022 \\
\hline 4 & PMM & 296.9622 & -94.3121 & -112.6822 \\
\hline 5 & MPP & -162.6422 & 353.3839 & 247.0022 \\
\hline 6 & MPM & -162.6422 & 353.3839 & -112.6822 \\
\hline 7 & MMP & -162.6422 & -94.3121 & 247.0022 \\
\hline 8 & MMM & -162.6422 & -94.3121 & -112.6822 \\
\hline
\end{tabular}

\section{Singularity analysis based on SKCs}

The singular configurations of a robotic mechanism corresponds to the rank deficiency of its Jacobian matrix, which means that the manipulator reaches a singularity when the determinant of its forward (A) or/and the determinant of its inverse (B) Jacobian matrix is (are) equal to zero ${ }^{[42][43]}$. Thus, three types of singularities can be determined accordingly:

- $\operatorname{det}(\mathbf{B})=0$, the robot loses one or more DOF and reaches a serial singularity

- $\operatorname{det}(\mathbf{A})=0$, the robot gains one or more uncontrolled DOF and encounters a parallel singularity

- $\operatorname{det}(\mathbf{A})=\operatorname{det}(\mathbf{B})=0$, the robot is in a configuration of mixed singularity. Here, we only focus on the first two types of singularities, as this Type of singularities can be obtained from the two previous ones.

\subsection{Singular configurations of $\mathrm{SKC}_{1}$}

From the previous section, $\mathrm{SKC}_{1}$ consists of loop 1, namely,

$$
\operatorname{LOOP}_{1}\left\{-\mathrm{P}_{11} \perp \mathrm{R}_{12}\left\|\mathrm{R}_{13}-\mathrm{Pa}_{2}\right\| \mathrm{P}_{21}-\right\}
$$

In order to solve the Jacobian matrix in $\mathrm{SKC}_{1}$, a position constraint equation is to be established. In this way, point $\mathrm{S}$ is the output point of the end-effector of $\mathrm{SKC}_{1}$. With known $S=\left[x_{S}, y_{S}, 0\right]^{T}$, the coordinates of points $\mathrm{C}_{1}$ and $\mathrm{C}_{2}$ are respectively expressed as

$$
C_{1}=\left[x_{S}+l_{13}, y_{S}, 0\right]^{T}, \quad C_{2}=\left[x_{S}, y_{S}+l_{23}, 0\right]^{T}
$$

Sequentially, the coordinates of points $\mathrm{B}_{1}$ and $\mathrm{B}_{2}$ are solved from equation (10). Through the rod length constraint $B_{1} C_{1}=l_{12}$ and $B_{2} C_{2}=l_{22}$, two position constraint equations for the $\mathrm{SKC}_{1}$ can be obtained

$$
\left\{\begin{array}{l}
\left(x_{B 1}-x_{C 1}\right)^{2}+\left(y_{B 1}-y_{C 1}\right)^{2}=l_{12}^{2} \\
\left(x_{B 2}-x_{C 2}\right)^{2}+\left(y_{B 2}-y_{C 2}\right)^{2}=l_{22}^{2}
\end{array}\right.
$$

Differentiating Eq. (22) with respect to time, the relationship between the output speed $\notin=\left[\begin{array}{ll}\& & \&\end{array}\right]^{T}$ of point $\mathrm{S}$ on the sub moving platform 1 in $\mathrm{SKC}_{1}$ and the active input speed $1 \&=\left[\begin{array}{ll}\& \& & \&\end{array}\right]^{T}$ is obtained as

$$
A \& \&+B \&=0
$$

with

$$
\begin{array}{r}
A=\left[\begin{array}{ll}
x_{B 1}-x_{C 1} & y_{B 1}-y_{C 1} \\
x_{B 2}-x_{C 2} & y_{B 2}-y_{C 2}
\end{array}\right], \quad B=\left[\begin{array}{cc}
g_{11} & 0 \\
0 & g_{22}
\end{array}\right] \\
g_{11}=-\left(x_{B 1}-x_{C 1}\right), \quad g_{22}=-\left(y_{B 2}-y_{C 2}\right)
\end{array}
$$

Now the singular configurations of $\mathrm{SKC}_{1}$ can be found in terms of the two following types..

(1)Serial singularity

When the serial singularity occurs, that is, $\operatorname{det}(\mathbf{B})=g_{11} g_{22}=0$, which means that at least one of diagonal elements in matrix $\mathbf{B}$ is equal to zero.

a) $g_{11}=-\left(x_{B 1}-x_{C 1}\right)=0$

That is, the $x$-coordinate of point $\mathrm{B}_{1}$ equals to that of point $C_{1}$. This means that link $B_{1} C_{1}$ is perpendicular to the guide rail (I), as shown in Fig. 10.

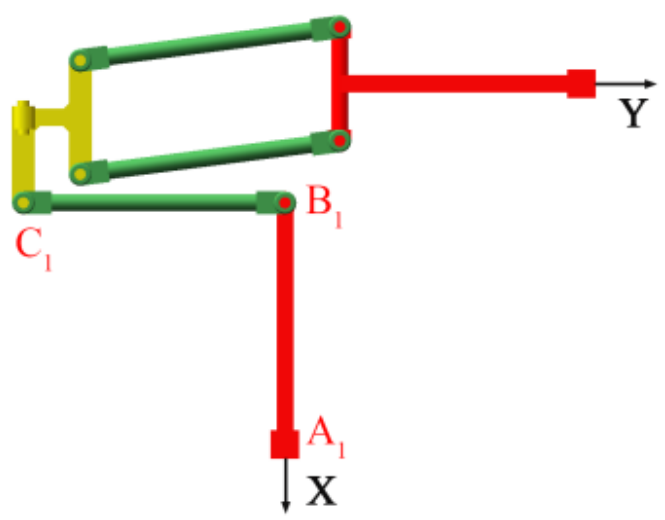

Fig.10 Serial singularity in $\mathrm{SKC}_{1}$

b) $g_{22}=-\left(y_{B 2}-y_{C 2}\right)=0$

That is, the $y$-coordinate of point $\mathrm{B}_{2}$ is equal to that of point $C_{2}$. In this case, link $B_{2} C_{2}$ is perpendicular to the guide rail (II), leading to the collinear parallelogram, which means that this singularity does not occur in practice due to the mechanical collision.

(2)Parallel singularity

For the convenience of analysis, matrix $\mathbf{A}$ is regarded as a matrix consisting of two row vectors, namely, $A=\left[\begin{array}{ll}\boldsymbol{e}_{1} & \boldsymbol{e}_{2}\end{array}\right]^{\mathrm{T}}$. When the parallel 
singularity occurs in the mechanism, i.e., $\operatorname{det}(\mathbf{A})=0$, vectors $\boldsymbol{e}_{1}$ and $\boldsymbol{e}_{2}$ are linearly dependent, namely,

$$
\boldsymbol{e}_{1}=k_{1} \boldsymbol{e}_{2} \text { or } \frac{y_{B 1}-y_{C 1}}{x_{B 1}-x_{C 1}}=\frac{y_{B 2}-y_{C 2}}{x_{B 2}-x_{C 2}}
$$

which means that links $C_{1} B_{1}$ and $C_{2} B_{2}$ are parallel in the XOY plane, as shown in Fig. 11.

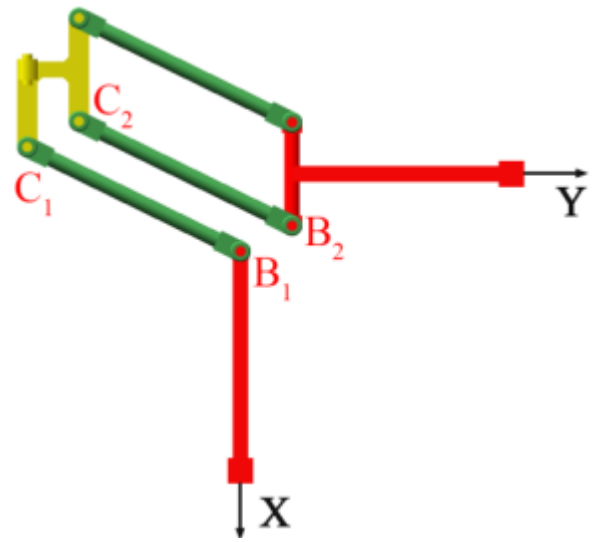

Fig.11 Parallel singularity in $\mathrm{SKC}_{1}$

\subsection{Singular configurations of $\mathrm{SKC}_{2}$}

Similarly, $\mathrm{SKC}_{2}$ is depicted as,

$$
\text { SOC }\left\{-\mathrm{Pa}_{(1)}^{(4 \mathrm{R})}-\mathrm{R}_{33}\left(\perp \mathrm{Pa}_{(3)}^{(4 \mathrm{R})}\right)\left\|\mathrm{R}_{32}\right\| \mathrm{P}_{31}-\right\}
$$

Analog to previous analysis, three position constraint equations for $\mathrm{SKC}_{2}$ is derived as

$$
\left\{\begin{array}{l}
x_{T}=x_{S} \\
\left(y_{S}-y_{T}\right)^{2}+\left(z_{S}-z_{T}\right)^{2}=l_{4}^{2} \\
\left(x_{B 3}-x_{C 3}\right)^{2}+\left(y_{B 3}-y_{C 3}\right)^{2}+\left(z_{B 3}-z_{C 3}\right)^{2}=l_{8}^{2}
\end{array}\right.
$$

Upon which, the relationship between the output speed $\& \&=\left[\begin{array}{lll}\& & \& & \&\end{array}\right]^{T}$ of point $\mathrm{P}$ on the end of moving platform and the input speed $1 \&=\left[\begin{array}{lll}\& & \& & \&\end{array}\right]^{T}$ of the active joints in the $\mathrm{SKC}_{2}$ is expressed as

$$
C \& 2+D \&=0
$$

where

$$
C=\left[\begin{array}{ccc}
1 & 0 & 0 \\
0 & y_{S}-y_{T} & z_{S}-z_{T} \\
x_{B 3}-x_{C 3} & y_{B 3}-y_{C 3} & z_{B 3}-z_{C 3}
\end{array}\right]
$$

$$
D=\left[\begin{array}{ccc}
1 & 0 & 0 \\
0 & y_{S}-y_{T} & 0 \\
0 & 0 & z_{B 3}-z_{C 3}
\end{array}\right]
$$

(1) Serial singularity

From $\operatorname{det}(\mathbf{D})=0$, serial singularity occurs when $z_{B 3}-z_{C 3}=0$ or/and $y_{S}-y_{T}=0$. In such two cases, all the links in the parallelogram will be collinear, which means that the serial singularity will not occur in practice due to the mechanical collision.

(2)Parallel singularity

The forward kinematic singularity occurs in $\mathrm{SKC}_{2}$, when $\operatorname{det}(\mathbf{C})=0$. Matrix $\mathbf{C}$ is regarded as a matrix consisting of three row vectors, namely, $C=\left[\begin{array}{lll}\boldsymbol{e}_{3} & \boldsymbol{e}_{4} & \boldsymbol{e}_{5}\end{array}\right]^{T}$, thus, singularity occurs when the two vectors $\boldsymbol{e}_{4}$ and $\boldsymbol{e}_{5}$ are linearly dependent, namely,

$$
\boldsymbol{e}_{4}=k_{2} \boldsymbol{e}_{5} \text { or } \frac{y_{B 3}-y_{C 3}}{x_{B 3}-x_{C 3}}=\frac{y_{S}-y_{T}}{x_{S}-x_{T}}
$$

which means that the projections of the links $\mathrm{B}_{3} \mathrm{C}_{3}$ and $\mathrm{ST}$ in the XOY plane are parallel, as depicted in Fig. 12.

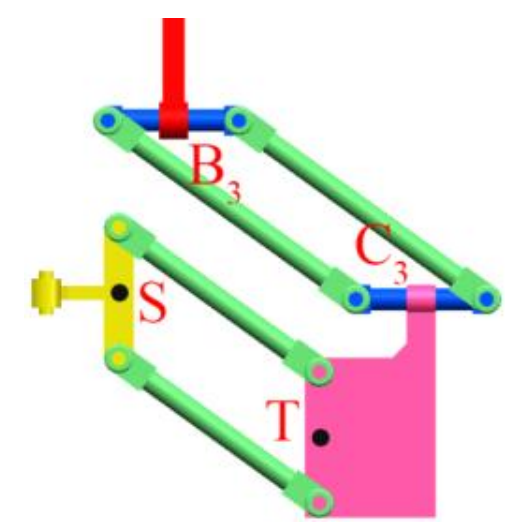

Fig. 12 Parallel singularity in $\mathrm{SKC}_{2}$

In this section, singularity analysis is carried out analytically and graphically. Two types of singularities are identified, which will be integrated in the following section for the workspace analysis, in order to find the singularity-free workspace. 


\section{Workspace analysis based on SKCs}

According to the principle of SKC-based mechanism composition and superposition of sub-workspaces, the workspace of the mechanism is to be found by two steps: (1) $\mathrm{SKC}_{1}$ generates the cross-section of the workspace; (2) $\mathrm{SKC}_{2}$ extends the workspace cross-section to forms the $3 \mathrm{D}$ volume subject to the constraints of $\mathrm{SKC}_{1}$.

\subsection{Find the workspace determined by $\mathrm{SKC}_{1}$}

6.1.1 Constraints on workspace

(1) Strokes of the active P-joints

For the $\mathrm{SKC}_{1}$ sub-parallel mechanism, the active joints have a limited motion ranges as follows

$$
\rho_{\text {min }} \leq \rho_{i} \leq \rho_{\text {max }},(i=1,2)
$$

where $\rho_{\min }$ and $\rho_{\max }$ represent the minimum and maximum strokes of the $i$ th active P-joint, respectively, and $\rho_{\min }=200, \rho_{\max }=600$.

(2) Motion ranges of the R-joints

As shown in Fig. 4, all the passive joints of the $\mathrm{SKC}_{1}$ sub-parallel mechanism are R-joints. In this branch, the angle $\alpha$ show in Fig. 7(a) should be in the range $\left[0,180^{\circ}\right]$ to avoid the collision between the joint $R_{\mathrm{c} 2}$ and the connecting $\operatorname{rod} R_{12} R_{13}$. In addition, the angle $\gamma_{i}$ in the parallelogram (2) are subject to the following constraints

$$
\gamma_{\text {min }} \leq \gamma_{i} \leq \gamma_{\max }
$$

and $\gamma_{\min }=-30^{\circ}, \gamma_{\max }=30^{\circ}$.

(3) Mechanical collision among the links

The mechanical collision may occur between the link $R_{12} R_{13}$ in the branch 1 and the link $R_{b 2} R_{c 2}$ in the branch 2 , which can be evaluated by the distance of the $\mathrm{R}$-joint $\mathrm{R}_{12}$ and link $\mathrm{R}_{\mathrm{b} 2} \mathrm{R}_{\mathrm{c} 2}$ below

$$
d_{i}>d
$$

where $d_{i}$ is the distance between the R-joint $\mathrm{R}_{12}$ and $\mathrm{R}_{\mathrm{b} 2} \mathrm{R}_{\mathrm{c} 2}$, and $d=12$.

6.1.2 Workspace identification

Since the mechanism has an analytic forward position equation, Eq. (14) can be deployed to identify the workspace and singularity loci. Figure 9 shows the workspace determined by $\mathrm{SKC}_{1}$, wherein the two different areas correspond to the two forward solutions. The serial singularity loci appears at the workspace boundaries, while the parallel singularity loci form a curve to divide the two workspace regions.

\subsubsection{Task workspace}

For the application of parallel mechanisms, a task workspace is defined as a regular geometry such as square or circle for 2-dimention and cuboid or cylinder for 3-dimention, which is helpful for robot programming. Accordingly, the regular area $300 \mathrm{~mm} \times 300 \mathrm{~mm}$ is identified as the task workspace as depicted in Fig. 13. Hence, the motion range of point $\mathrm{S}$ in brank $\mathrm{SKC}_{2}$ is expressed as

$$
-140 \leq x_{S} \leq 160, \quad 150 \leq y_{S} \leq 150
$$

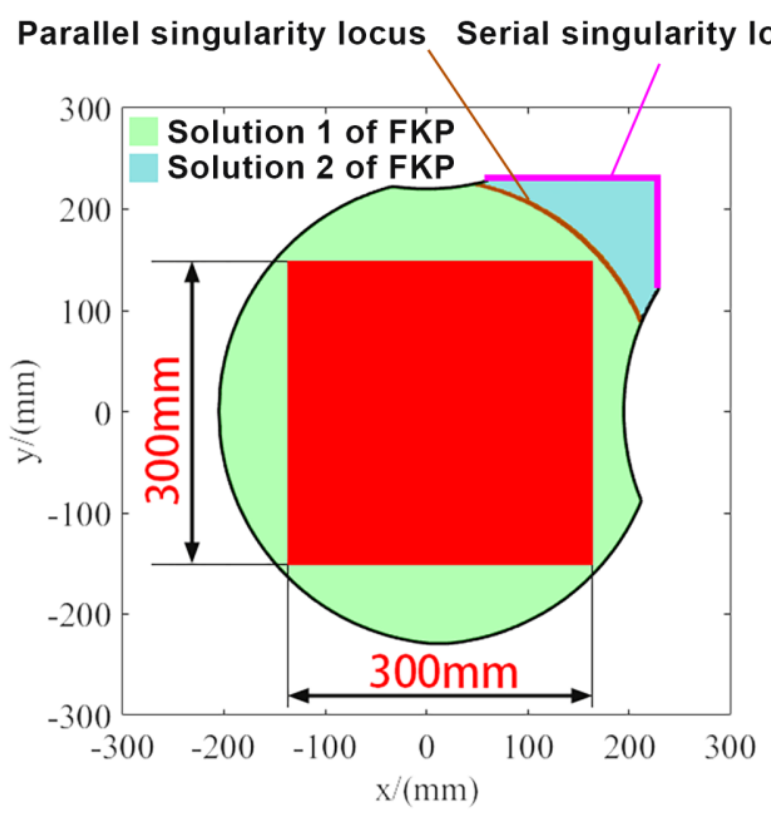

Fig.13 Workspace determined by $\mathrm{SKC}_{1}$

\subsection{Workspace determined by $\mathrm{SKC}_{2}$}

The workspace cross-section, namely, the project of the boundaries in $X Y$ plane, is determined by $\mathrm{SKC}_{1}$, while the dimension of the workspace along $Z$-axis will be determined by $\mathrm{SKC}_{2}$.

6.2.1 Constraints on workspace

(1) Stroke of active P-joint

For $\mathrm{SKC}_{2}$, the constraints on the motion range include the output link of $\mathrm{SKC}_{1}$ expressed in Eq. (29) and the stroke of active $\mathrm{P}$-joint $\rho_{3}$ in $\mathrm{SKC}_{2}$ below

$$
\rho_{\text {min }} \leq \rho_{3} \leq \rho_{\text {max }}
$$

where $\rho_{\text {min }}=230, \rho_{\text {max }}=570$.

(2) Motion range of R-joint

As shown in Fig. 4(a), the R-joints in parallelograms (1) and (3) has the following constraint

$$
\gamma_{\text {min }} \leq \gamma_{j} \leq \gamma_{\max }
$$

where $\gamma_{\min }=-30^{\circ}$ and $\gamma_{\max }=30^{\circ}$.

(3) Mechanical collision

With the previous constraints, mechanical collision will not occur between the hybrid branch 2 and parallelograms in $\mathrm{SKC}_{2}$.

6.2.2 Reachable workspace

Subject to the previous kinematic constraints, the workspace determined by $\mathrm{SKC}_{2}$ as well as $\mathrm{SKC}_{1}$ is 
shown in Fig. 14.

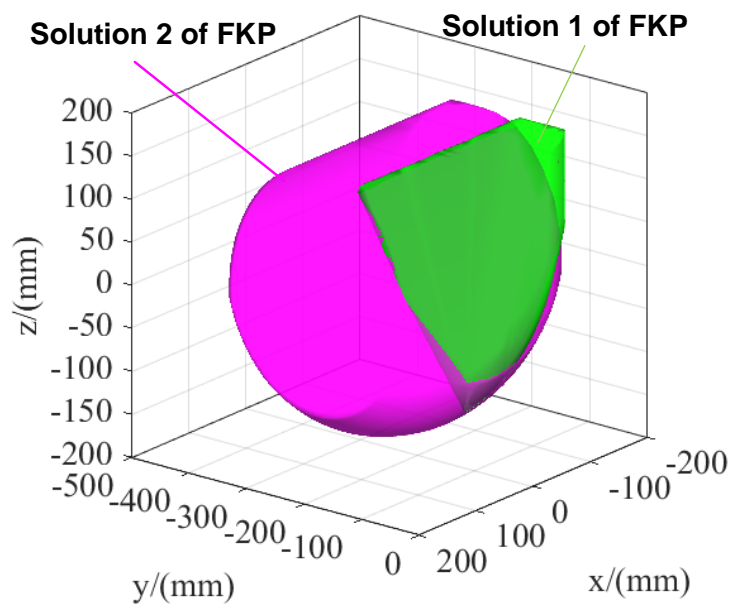

Fig.14 Maximum workspace by the $\mathrm{SKC}_{1}$ and $\mathrm{SKC}_{2}$

By means of the Gröbner-based elimination method $^{[44][45]}$, the serial and parallel singular loci of $\mathrm{SKC}_{2}$ can be identified with Maple ${ }^{\mathrm{TM}}$, which are displayed in Fig. 15. It can be observed that the serial singularity loci forms a closed volume in the shape of cylinder, and the parallel singularity loci are located inside. Consequently, a singularity-free workspace can be selected accordingly.

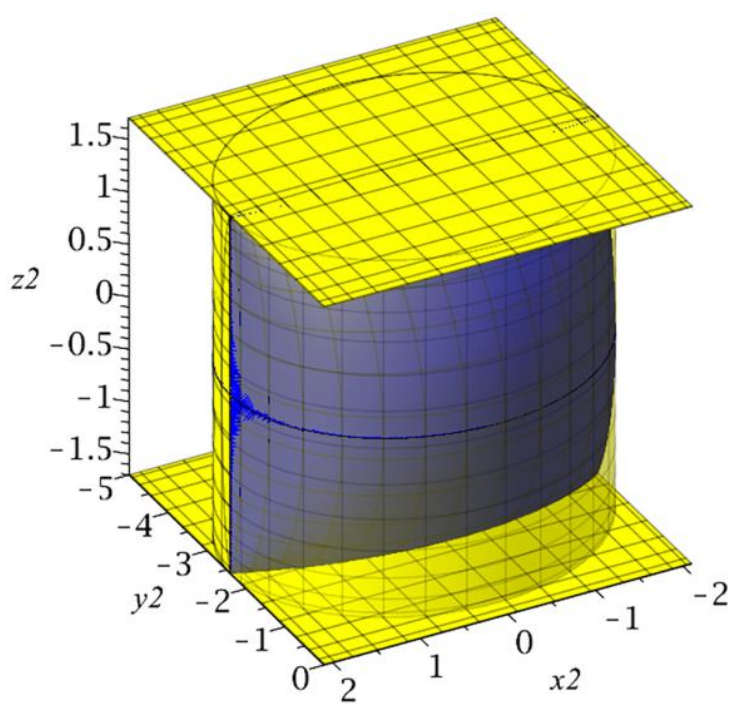

Fig.15 Serial (yellow surface) and parallel (blue surface) singularity loci of $\mathrm{SKC}_{2}$

To depict the relationship between the workspace and singularity loci by $\mathrm{SKC}_{2}, \mathrm{a}$ combination of Figs. 14 and 15 is made as shown in Fig. 16. It is seen that the most volume of the workspace shown in Fig. 16 are bounded by the surface generated by the parallel singularity loci, which means that a large envelop can be found as the singularity-free workspace.

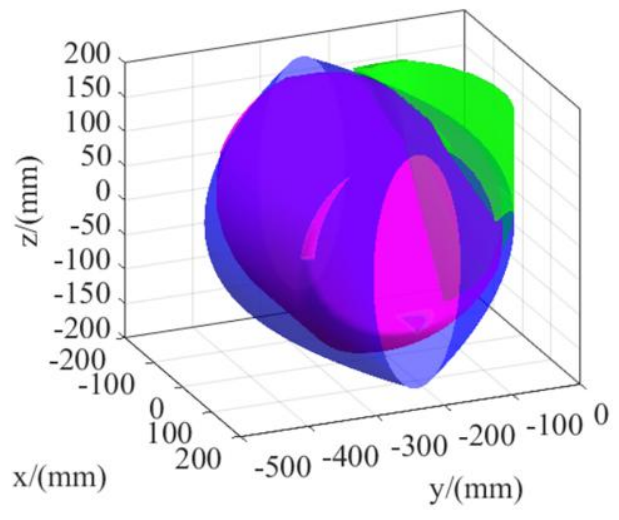

(a)

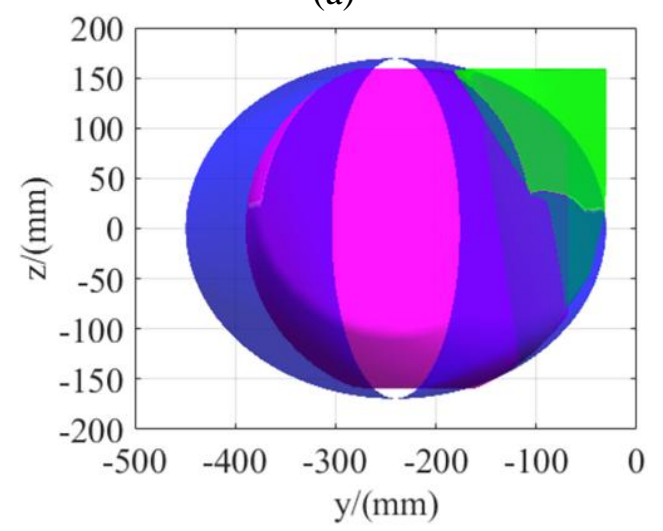

(b)

Fig.16 Workspace (pinkish) and parallel singular loci (blue) by $\mathrm{SKC}_{2}$ : (a) isometric view; (b) side view normal to $X$-axis

\subsubsection{Task workspace}

Based upon the previous workspace and singularity analysis, Fig. 17 shows an internal cuboid with side lengths $280 \times 200 \times 180 \mathrm{~mm}^{3}$ as the task workspace free of singularity. For a better view, the task and reachable workspaces are displayed in Fig. 18.

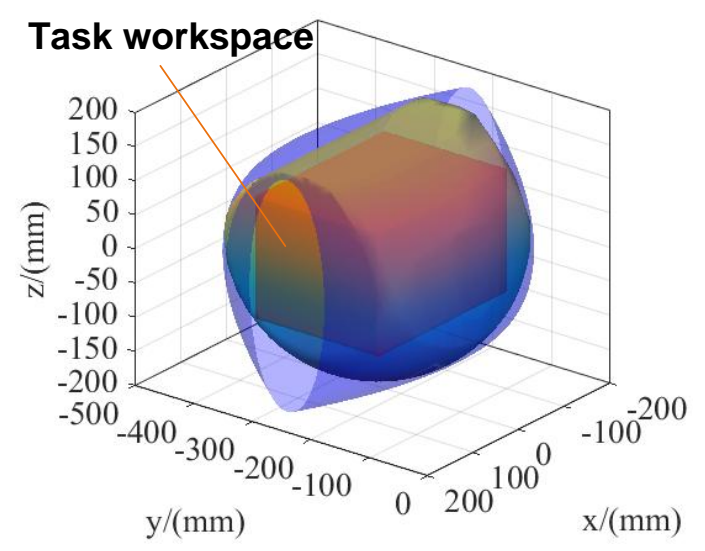

Fig.17 Singularity-free task workspace inside the reachable workspace 


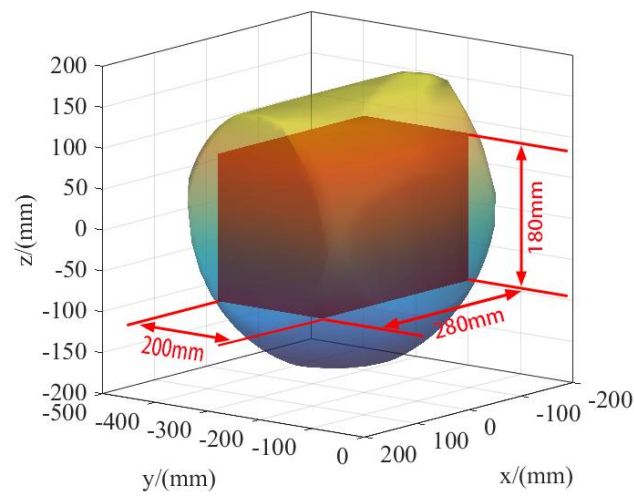

(a)

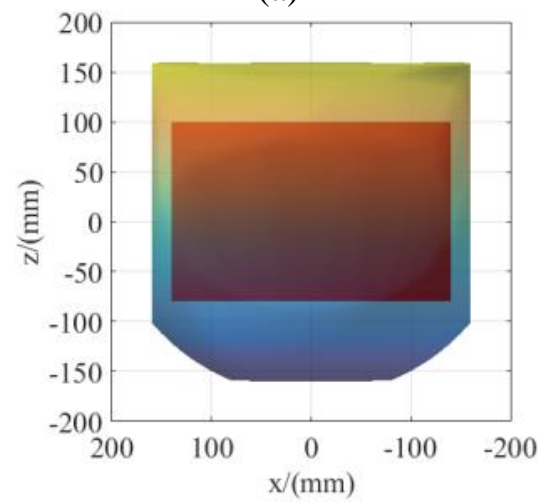

(b)

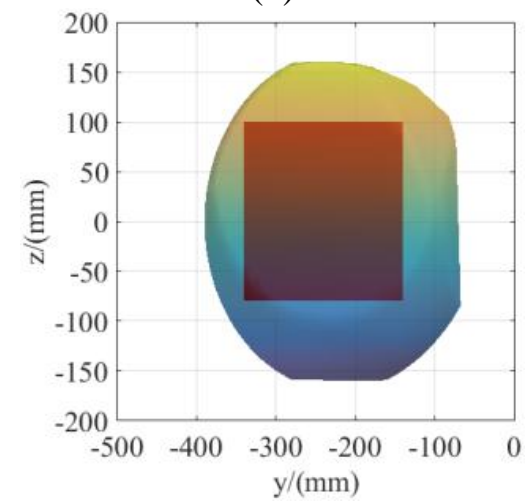

(c)

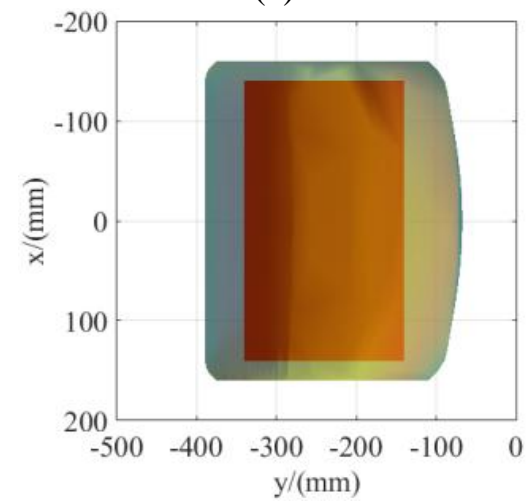

(d)

Fig.18 Task workspace inside the reachable workspace of $\mathrm{SKC}_{2}$ : (a) isometric view; (b), (c), (d) side views

The Vari-Orthoglide parallel mechanism is designed based on the superposition principle of sub-workspace determined by the SKC units. Figure
19 shows the workspace cross-section with $Z$-coordinate equal to 0 , which is determined by $\mathrm{SKC}_{1}$ under the geometric constraints. The overall regular workspace can be obtained by stretching the rectangle $280 \times 200 \mathrm{~mm}^{2}$ along bidirectional $Z$-axis with $180 \mathrm{~mm}$, respectively, which is the designated task workspace.

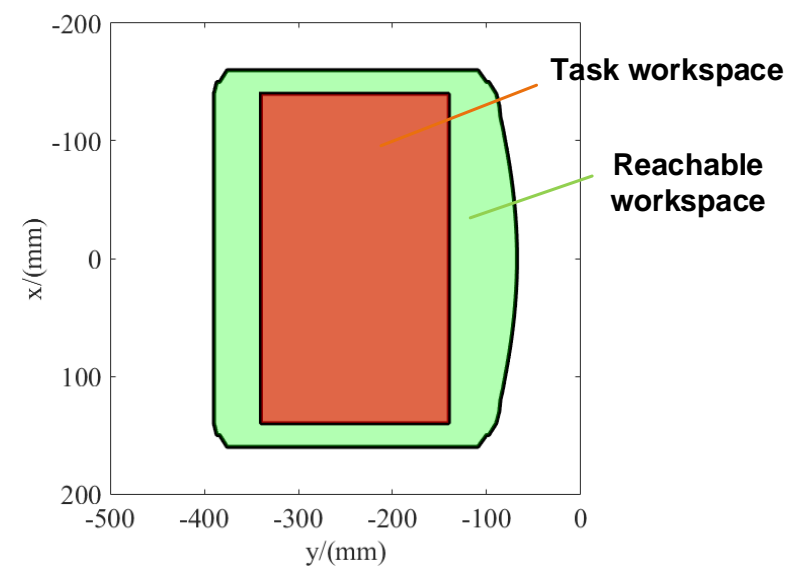

Fig. 19 Workspace cross-section with $Z=0$

\section{Conclusions}

In this paper, a new translational parallel mechanism named as Vari-Orthoglide is proposed. Based on the architectural topology analysis, it turns out that the coupling degree of the mechanism is equal to zero, which means the partially decoupled kinematics. The mechanism consists of two single kinematic chains to ease the forward kinematic modeling, leading to the analytical solutions. By making use of the forward kinematic solutions, the workspace of the mechanism corresponding to different assembly modes is found for the further performance evaluation. The advantages of the symbolic forward kinematics for workspace and singularity evaluation include: (1) higher efficiency and precision to find all the possible positions of the moving platform subject to the motion ranges of the active joints; (2) the mapping between the forward kinematics and workspace helpful to avoid singular configurations in the trajectory planning.

Using the Gröbner-based elimination method, all the serial and parallel singular loci of the proposed mechanism are identified, from which a regular workspace free of singularity is selected as the task workspace with large volume. With the architectural topological characteristics analysis, workspace evaluation and singularity identification, this paper presents a step-design approach by integrating the kinematic decoupling and superposition of sub-workspace determined by the single kinematic chains, applicable to other types of parallel mechanisms. 


\section{Acknowledgement}

The financial support from National Natural Science Foundation of China (No. 51975062) is gratefully acknowledged.

\section{References}

[1] Clavel R. Device for the movement and positioning of an element in space. U.S. Patent No. 4,976,582, 1990.

[2] Clavel R. Conception d'un robot parallèle rapide à quatre degrés de liberté. PhD Thesis \#925, EPFL, Ch-Lausanne, 1991.

[3] ABB Robotics. IRB 360 FlexPicker: https://new.abb.com/products/robotics/industrialrob ots/irb-360, 1994. Accessed: 20.12.2019

[4] Stock M, Miller K. Optimal kinematic design of spatial parallel manipulators: application to linear delta robot. ASME J. Mech. Des., 2003, 125(2):292-301.

[5] Bouri M, Clavel R, Zerrouki MY, Maeder W. Towards a new Delta robot: an inverted Delta. In Proceedings of the International Symposium of Robotics, Paris 2004.

[6] Bouri M, Clavel R. The linear delta: Developments and applications. In ISR 2010 (41st International Symposium on Robotics) and ROBOTIK 2010 (6th German Conference on Robotics) 2010 Jun 7 (pp. 1-8). VDE.

[7] Kelaiaia R, Company O, Zaatri A. Multiobjective optimization of a linear Delta parallel robot. Mech. Mach. Theory. 2012, 50:159-178.

[8] Mazare M, Taghizadeh M, Najafi MR. Kinematic analysis and design of a 3-DOF translational parallel robot. Int. J. Autom. Comput., 2017, 14(4):432-441.

[9] Shen H, Wang Y, Wu G, Meng Q. Stiffness Analysis of a Semi-symmetrical Three-Translation Delta-CU Parallel Robot. In IFToMM International Symposium on Robotics and Mechatronics, 2019 Oct 28 (pp. 3-15). Springer, Cham.

[10] Kong X, Gosselin CM. Type synthesis of 3-DOF translational parallel manipulators based on screw theory. ASME J. Mech. Des., 2004, 126(1):83-92.

[11] Gogu G. Structural synthesis of fully-isotropic translational parallel robots via theory of linear transformations. Euro. J. Mechanics-A/Solids, 2004, 23(6):1021-1039.

[12] Carricato M, Parenti-Castelli V. A family of 3-DOF translational parallel manipulators. ASME J. Mech. Des., 2003, 125(2):302-7.

[13] Lee CC, Hervé JM. Translational parallel manipulators with doubly planar limbs. Mech. Mach. Theory, 2006, 41(4):433-55.
[14] Tsai LW, Joshi S. Kinematics and optimization of a spatial 3-UPU parallel manipulator. ASME J. Mech. Des., 2000, 122(4):439-46.

[15] Di Gregorio R, Parenti-Castelli V. Mobility analysis of the 3-UPU parallel mechanism assembled for a pure translational motion. ASME J. Mech. Des. 2002, 124(2):259-64.

[16] Corves B, Brinker J, Lorenz M, Wahle M. Design methodology for translational parallel manipulators exhibiting actuation redundancy. Proc. Ins. Mech. Eng. Part C J. Mech. Eng. Sci., 2016,230(3):425-36.

[17] Bhutani G, Dwarakanath TA. Novel design solution to high precision 3 axes translational parallel mechanism. Mech. Mach. Theory. 2014, 75:118-30.

[18] Toz M, Kucuk S, Dimensional optimization of 6-DOF 3-CCC type asymmetric parallel manipulator Adv. Robot., 2014, 28(9): 625-637.

[19] Di Gregorio R. Kinematics of the translational 3-URC mechanism. ASME J. Mech. Des., 2004, 126(6):1113-7.

[20] Li B, Li YM, Zhao XH, Ge WM. Kinematic analysis of a novel 3-CRU translational parallel mechanism. Mech. Sci., 2015, 6(1):57-64.

[21] Li Y, Xu Q. Kinematic Analysis and Design of a New 3-DOF Translational Parallel Manipulator. ASME. J. Mech. Des, 2006, 128(4): 729-737.

[22] Xu Q, Li Y. Design and analysis of a new singularity-free three-prismatic-revolute-cylindrical translational parallel manipulator. Proc. Ins. Mech. Eng. Part C J. Mech. Eng. Sci., 2007,221(5):565-76.

[23] Zeng Q, Ehmann KF, Cao J. Tri-pyramid Robot: Design and kinematic analysis of a 3-DOF translational parallel manipulator. Robot. Comp.-Int. Manuf., 2014, 30(6):648-657.

[24] Kong X, Gosselin CM. Kinematics and singularity analysis of a novel type of 3-CRR 3-DOF translational parallel manipulator. Int. J. Robot. Res., 2002, 21(9):791-798.

[25] Briot S, Bonev IA. Pantopteron: a new fully decoupled 3DOF translational parallel robot for pick-and-place applications. ASME J. Mech. Robot., 2009, 1(2): 021001.

[26] Carricato M, Parenti-Castelli V. Singularity-free fully-isotropic translational parallel mechanisms. Int. J. Robot. Res., 2002, 21(2):161-174.

[27] Chablat D, Wenger P. Architecture optimization of a 3-DOF translational parallel mechanism for machining applications, the Orthoglide. IEEE Trans. Robot. Autom., 2003, 19(3):403-410.

[28] Lou Y, Li Z. A novel 3-DOF purely translational parallel mechanism. In IEEE/RSJ Int. Conf. Intell. Robot. Syst., 2006 Oct 9 (pp. 2144-2149). IEEE. 
[29] Wu G, Shen H. Parallel PnP Robots, Research on Intelligent Manufacturing, vol. 7, Springer, Singapore, 2021.

[30] Hay AM, Snyman JA. Methodologies for the optimal design of parallel manipulators. Int. J. Numer. Meth. Eng. . 2004, 59(1):131-152.

[31] Lou Y, Liu G, Chen N, Li Z. Optimal design of parallel manipulators for maximum effective regular workspace. In IEEE/RSJ Int. Conf. Intell. Robot. Syst. (pp. 795-800). Edmonton, Alta., Canada, 2005.

[32] Bulca F, Angeles J, Zsombor-Murray PJ. On the workspace determination of spherical serial and platform mechanisms. Mech. Mach. Theory, 1999, 34(3):497-512.

[33] Briot S, Pashkevich A, Chablat D. Optimal technology-oriented design of parallel robots for high-speed machining applications. In IEEE Int. Conf. Robot. Autom., 2010 May 3 (pp. 1155-1161).

[34] Wu G, Caro S, Bai S, Kepler J. Dynamic modeling and design optimization of a 3-DOF spherical parallel manipulator. Robot. Auto. Syst., 2014, 62(10):1377-1386.

[35] Cammarata A. Optimized design of a large-workspace 2-DOF parallel robot for solar tracking systems. Mech. Mach. Theory. 2015, 83:175-86.

[36] Kaloorazi MH, Masouleh MT, Caro S. Determination of the maximal singularity-free workspace of 3-DOF parallel mechanisms with a constructive geometric approach. Mech. Mach. Theory, 2015, 84:25-36.

[37] Kucuk S. Dexterous Workspace Optimization for a New Hybrid Parallel Robot Manipulator. ASME. J. Mech. Robot., 2018; 10(6): 064503.

[38] Yang TL, Liu A, Shen H, Hang L, Luo Y, Jin Q. Topology Design of Robot Mechanisms. Springer Singapore, 2018.

[39] Damien Chablat, Philippe Wenger, Félix Majou. The Optimal Design of Three Degree-of-Freedom Parallel Mechanisms for Machining Applications. In IEEE Int. Conf. Robot. Autom., 2003, Coimbra, Portugal, IEEE, pp.1-6.

[40] Gao X-S, Lei D, Liao Q, Zhang G-F. Generalized Stewart-Gough platforms and their direct kinematics. IEEE Trans. Robot., 2005, 21(2): 141-151.

[41] Shen H, Yang TL, Li J, Zhang D, Deng J, Liu A. Evaluation of topological properties of parallel manipulators based on the topological characteristic indexes. Robotica., 2020, 38(8):1381-1399.

[42] Gosselin C, Angeles J. Singularity analysis of closed-loop kinematic chains. IEEE Trans. Robot. Autom., 1990, 6(3): 281-290.
[43] Wu G, Bai S, Caro S. A transmission quality index for a class of four-limb parallel Schönflies motion generators. ASME J. Mech. Robot., 2018, 10(5): 051014.

[44] Shen H, Chablat D, Zeng B, Li J, Wu G, Yang TL. A Translational Three-Degrees-of-Freedom Parallel Mechanism With Partial Motion Decoupling and Analytic Direct Kinematics. ASME J. Mech. Robot., 2020, 12(2): 021112.

[45] Chablat D, Wenger P, Merlet J. Workspace analysis of the orthoglide using interval analysis. In Advances in Robot Kinematics 2002 (pp. 397-406). Springer, Dordrecht. 